\title{
Primeros aportes sobre variaciones en la abundancia de Jaguar (Panthera onca) en el río Bita (Puerto Carreño, Vichada)
}

\author{
Germán Garrote \\ Fundación Omacha, Bogotá D.C., Colombia \& Instituto de Biología de la Conservación (IBICO), Torres de Albanchez, \\ España.gergarrote@gmail.com
}

La información disponible sobre los felinos de Colombia es muy escasa, lo que dificulta la toma de decisiones sobre su conservación (Arias-Alzate et al. 2013). El Jaguar (Panthera onca) está catalogado como Casi Amenazado a nivel mundial (Caso et al. 2008) y Vulnerable para Colombia (Rodríguez-Mahecha et al. 2006). A pesar de ser una de las especies de felino más estudiadas en Colombia (Pinilla-Buitrago et al. 2015), existe una gran carencia de información básica (e.g. presas potenciales) para la Orinoquia colombiana, tanto en áreas protegidas como en áreas no protegidas (Garrote 2008). Concretamente los Llanos Orientales colombianos son considerados área prioritaria para el estudio y conservación del Jaguar dado el escaso conocimiento sobre su presencia local y estado poblacional (Sanderson et al. 1999). Los trabajos sobre el Jaguar realizados en los Llanos Orientales son muy escasos, centrándose casi con exclusividad en el conflicto ganadero (Garrote 2012, Payan 2005).

En este estudio se evalúa la variación de la abundancia de Jaguar a lo largo del río Bita en el Municipio de Puerto Carreño (Vichada) mediante el uso de un índice de abundancia. Los índices de abundancia han sido utilizados ampliamente para evaluar la abundancia de mamíferos debido a que variaciones en los índices suelen reflejar variaciones en la densidades absolutas (Garrote et al. 2014).

El municipio de Puerto Carreño (Vichada) está situado en la confluencia de los ríos Meta y Orinoco $\left(06^{\circ} 11^{\prime} 16^{\prime}\right.$ ' N, $67^{\circ} 28^{\prime}$ $23^{\prime}$ 'W). Posee una elevación entre 50 y $100 \mathrm{msnm}$, una temperatura media de $28^{\circ} \mathrm{C}$ y una precipitación media anual de 2176 mm (IGAC 1996). El río Bita recorre de Oeste a Este el municipio de Puerto Carreño y desemboca en el río Orinoco a la altura de la Ciudad de Puerto Carreño. En época seca (diciembre-marzo) emergen numerosas playas de arena.

Entre el 23 de enero y el 16 de febrero de 2007 se recorrieron por agua tres tramos del río Bita en busca de la presencia de Jaguar (Figura 1): 1) Tramo alto, situado entre 75 y $115 \mathrm{~km}$ en línea recta de la desembocadura del río Bita con el Orinoco y misma distancia a la ciudad de Puerto Carreño. En este tramo se recorrieron $105 \mathrm{~km}$ continuos de río en cuatro días consecutivos, donde se seleccionaron 40 playas al azar que fueron recorridas en busca de huellas de la especie. 2) Tramo medio, situado entre 25 y $35 \mathrm{~km}$ en línea recta de la desembocadura. En este tramo se recorrieron 25 kilómetros de río en un único día, en las que todas las playas ( $\mathrm{n}=21)$ fueron muestreadas. Y 3) tramo bajo, situado entre 5 y $23 \mathrm{~km}$ en línea recta de la desembocadura donde se recorrieron $20 \mathrm{~km}$. Sin embargo, el número de playas emergidas adecuadas para el muestreo fue tan sólo de 4. El escaso número de playas muestreadas en este tramo hace que los resultados obtenidos no sean concluyentes, por lo que no fueron considerados. Se estimó como índice de abundancia de Jaguar el porcentaje de playas con presencia detectada respecto al total de las muestreadas en cada tramo.

En el tramo alto se detectaron huellas de Jaguar en 31 playas (77,5\%) mientras que en el tramo medio se detectaron huellas en 3 playas (14,3\%). A pesar de que en el tramo alto las playas muestreadas fueron tan solo una muestra de las existentes, y no la totalidad, como se realizó en el tramo medio, el esfuerzo de muestreo llevado a cabo ( $\mathrm{n}=40$ playas), es suficiente para considerarlo como representativos del tramo estudiado.

Mammalogy Notes | Notas Mastozoológicas

Sociedad Colombiana de Mastozoología

Vol. 2 Num. 1| 2015 


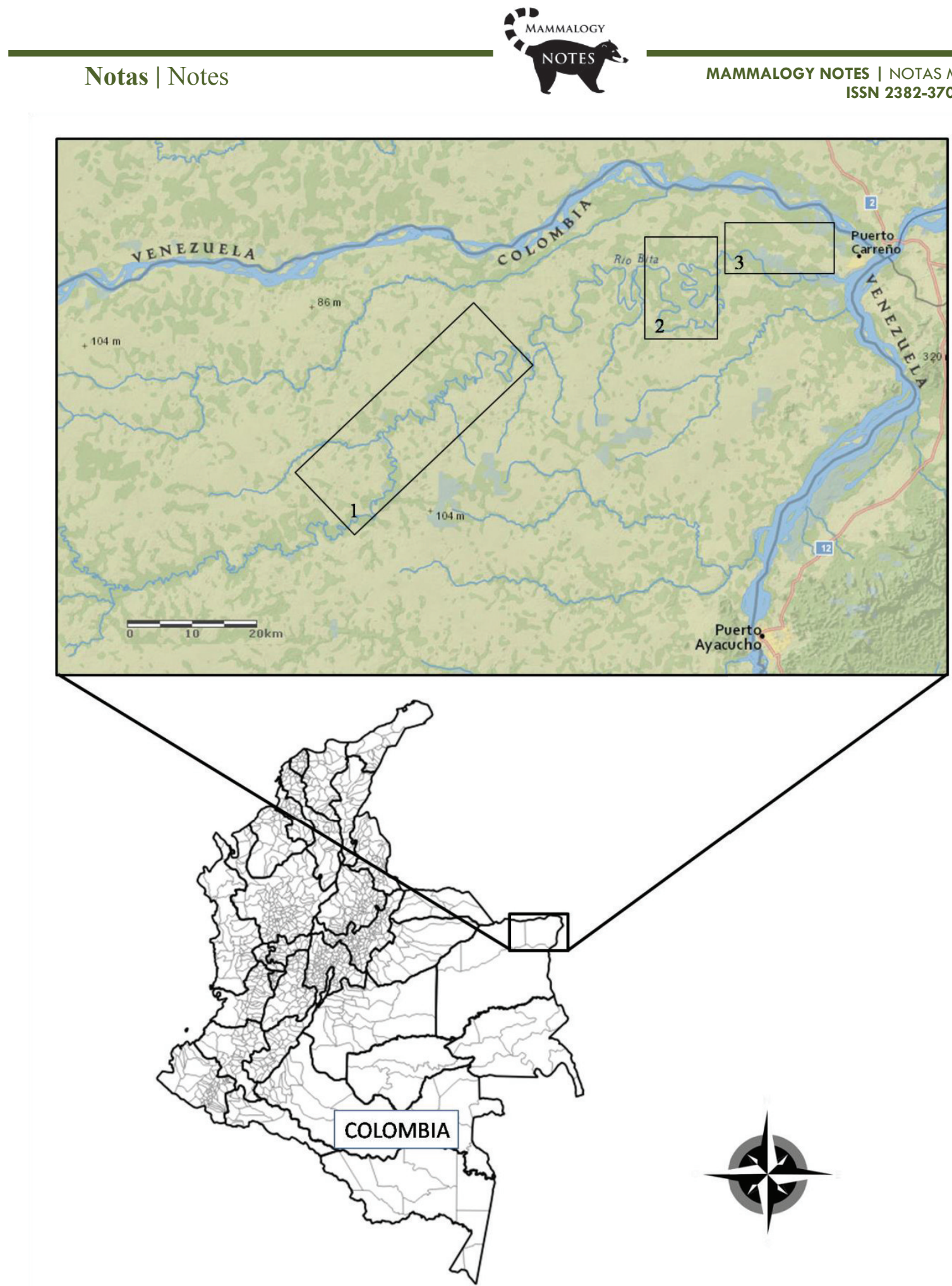

Figura 1. Área de estudio en los tres tramos del río Bita en el Municipio de Puerto Carreño (Vichada, Colombia). 1) tramo alto; 2) tramo medio y 3) tramo bajo.

Los resultados indican un notable incremento en la tasa de detección de Jaguar en el tramo más alto del río Bita. Por otro lado, Garrote \& Trujillo (2006), en un estudio realizado en la Reserva Bojonawi, situada en la orilla del río Orinoco a menos de 5 $\mathrm{km}$ de la desembocadura del río Bita, concluyeron que la presencia de Jaguar en el área era ocasional. Considerada en conjunto, la información disponible podría estar indicando la existencia de una mayor abundancia de Jaguar en el río Bita en tramos más alejados a la desembocadura con el río Orinoco y de la población de Puerto Carreño. Esto se corresponde con

\section{Mammalogy Notes | Notas Mastozoológicas \\ Sociedad Colombiana de Mastozoología \\ Vol. 2 Num. 1 | 2015}




\section{Notas | Notes}

resultados obtenidos en otros estudios en los que se observó una relación de abundancia de Jaguar inversa a la densidad humana (Woodroffe 2000), menor presencia de la especie en la cercanía de los pueblos (Payán 2009), y mayor riesgo de conflicto con los humanos en las cercanías a vías de comunicación, indicativas del grado de desarrollo humano (GonzalezMaya et al. 2012). La presencia humana implica la persecución directa del Jaguar y la reducción de sus presas (Zeller, 2007). A medida que se aleja de núcleos urbanos o grandes vías de comunicación, la densidad humana va disminuyendo y se va dificultando el acceso a zonas cada vez más remotas con malas comunicaciones y dificultad de transporte, lo que permite que se mantenga el área bien conservada (Payan 2005) como es el caso del río Bita que mantiene el 85-95\% de sus ecosistemas intactos (Romero et al. 2004).

A pesar de la sencillez de los resultados presentados en este estudio, tienen el valor de ser los primeros que apuntan importantes variaciones en abundancia de Jaguares en el río Bita a medida que se asciende en su cauce. Esta información preliminar puede ser tenida en cuenta en la creación y localización de nuevas áreas protegidas de cara a optimizar la conservación de las poblaciones del Jaguar y sus presas en la región. Sin embargo, sería deseable el desarrollo de futuros estudios que permitan conocer en profundidad hasta qué punto esta alta tasa de detección de la especie en tramos altos del río se corresponde con la existencia de poblaciones saludable de Jaguares y sus presas en la región.

\section{Agradecimientos}

Este estudio fue realizado en el marco del proyecto "Pijiwi Orinoko" Fundación Omacha-Fundación Horizonte Verde y Forest Conservation Agreement. Agradezco a Jacinto Terán y a Ives sin los cuales este trabajo no hubiera sido posible. Los comentarios de dos revisores anónimos mejoraron notablemente el manuscrito inicial.

\section{Referencias}

ARIAS-ALZATE, A., et al. 2013. Presencia de felinos y evidencias de conflicto con humanos en tres regiones de Antioquia. Pp. 145-154 in Grandes Felinos de Colombia, Vol I (Payán Garrido E \& Castaño-Uribe C eds.), Panthera Colombia, Fundación Herencia Ambiental Caribe, Conservación Internacional \& Cat Specialist Group UICN/SSC, Bogotá, Colombia.

CASO, A., et al. 2008. Panthera onca. The IUCN Red List of Threatened Species. Version 2015.1. <www.iucnredlist.org>. Downloaded on 17 June 2015.

IGAC. 1996. Diccionario geográfico de Colombia. Instituto Geográfico Agustín Codazzi, Ministerio de Hacienda. Santafé de Bogotá.

GARROTE, G. \& TRUjILlo F. 2006. Caracterización de la comunidad de carnívoros de la Reserva Natural Bojonawi (R.B. Tuparro, Vichada, Colombia).. II Congreso Colombiano de Zoología. Santa Marta, Noviembre 26 a Diciembre 1, Colombia.

GARROTE, G. 2008. El Jaguar (Panthera onca). Pp.65- 74, en: Plan de manejo y conservación de especies amenazadas en la Reserva de la Biosfera El Tuparro: Delfines de río, Manatíes, Nutrias, Jaguares y Tortugas del género Podocnemis (F Trujillo, M Portocarrero y G Gómez, eds.). Proyecto Pijiwi Orinoco (Fundación Omacha -Fundación Horizonte Verde), Forest Conservation Agreement, Bogota, Colombia.

GARROTE, G. 2012. Depredación del Jaguar (Panthera onca) sobre el ganado en los llanos orientales de Colombia. Mastozoología Neotropical 19: 139-145.

GARROTE, G., PEREZ DE AYALA, R., J.L. TELLERÍA. 2014. A comparison of scat counts and camera trapping to assess Iberian lynx abundance. European Journal of Wildlife Research. 60: 885-889.

GONZÁLEZ-MAYA, J.F., et al. 2012. Conflictos felinos-vida silvestre en el Caribe Colombiano: un estudio de caso en los departamentos del Cesar y La Guajira.Pp.51-59. En: Castaño-Uribe C, et al. (Eds). Plan de Conservación de Felinos del Caribe Colombiano: Los felinos y su papel en la planificación regional integral basada en especies clave.

PAYÁN, C. E. 2005. Jaguar conservation in the Colombian llanos: Presence, local perceptions and the livestock conflict. Annual report, 42 pp. Wildlife Conservation Society, New York, EE.UU.

PAYÁN, C. E. 2009. Hunting as sustainability, species richness and carnivore conservation in Colombian Amazonia. London University College. London.

PINILlA-BUitRAGO, G. E., et al. 2014. Familia Felidae. Pp: 78-103 en: Los carnívoros terrestres y semiacuáticos continentales de Colombia (Suárez-Castro, A.F. \& H.E. Ramírez-Chaves Eds). Editorial Universidad Nacional de Colombia, Bogotá, Colombia.

RODRÍGUEZ-MAHECHA, J., et al. 2006. Jaguar Panthera onca en: Libro rojo de los Mamíferos de Colombia. Serie libro rojos de especies amenazadas de Colombia (RodríguezMahecha, J., et al. Eds). Conservación Internacional Colombia, Ministerio de Ambiente, Vivienda y Desarrollo Territorial, Bogotá, Colombia.

ROMERO, M., et al. 2004. Ecosistemas de la cuenca del Orinoco colombiano. Instituto Alexander von Humbolt. Bogotá, Colombia.

WOODROFFE, R. 2000. Predators and people: using densities to interpret declines of large carnivores. Animal Conservation 3: 165-173.

ZELLER K. 2007. Jaguar in the New Millenium Data Set Update: The State of Jaguar in 2006. Wildlife Conservation Society's Jaguar Conservation Program. 